\title{
Evaluating the Effectiveness of Cryopreserved Acellular Dermal Matrix in Immediate Expander- Based Breast Reconstruction: A Comparison Study
}

\author{
So-Young Kim, So Young Lim, Goo-Hyun Mun, Sa-Ik Bang, Kap Sung Oh, Jai-Kyong Pyon \\ Department of Plastic Surgery, Samsung Medical Center, Sungkyunkwan University School of Medicine, Seoul, Korea
}

Background CGCryoDerm was first introduced in 2010 and offers a different matrix preservation processes for freezing without drying preparation. From a theoretical perspective, CGCryoDerm has a more preserved dermal structure and more abundant growth factors for angiogenesis and recellularization. In the current study, the authors performed a retrospective study to evaluate freezing- and freeze-drying-processed acellular dermal matrix (ADM) to determine whether any differences were present in an early complication profile.

Methods Patients who underwent ADM-assisted tissue expander placement for two stage breast reconstruction between January of 2013 and March of 2014 were retrospectively reviewed and divided into two groups based on the types of ADM-assisted expander reconstruction (CGDerm vs. CGCryoDerm). Complications were divided into four main categories and recorded as follows: seroma, hematoma, infection, and mastectomy skin flap necrosis.

Results In a total of 82 consecutive patients, the CGCryoDerm group had lower rates of seroma when compared to the CGDerm group without statistical significance (3.0\% vs. $10.2 \%$, $P=0.221$ ), respectively. Other complications were similar in both groups. Reconstructions with CGCryoDerm were found to have a significantly longer period of drainage when compared to reconstructions with CGDerm (11.91 days vs. 10.41 days, $\mathrm{P}=0.043$ ).

Conclusions Preliminary findings indicate no significant differences in early complications between implant/expander-based reconstructions using CGCryoderm and those using CGDerm.

Keywords Acelluar dermis / Tissue expander devices / Seroma
Correspondence: Jai-Kyong Pyon Department of Plastic and Reconstructive Surgery, Samsung Medical Center, Sungkyunkwan University School of Medicine, 81 Irwon-ro, Gangnam-gu, Seoul 135-710, Korea Tel: $+82-2-3410-2236$ Fax: +82-2-3410-0036 E-mail: psbboy@gmail.com

No potential conflict of interest relevant to this article was reported.

Received: 8 Oct 2014 • Revised: 22 Nov 2014 • Accepted: 3 Dec 2014

pISSN: 2234-6163 • elSSN: 2234-6171 • http://dx.doi.org/10.5999/aps.2015.42.3.316 • Arch Plast Surg 2015;42:316-320

\section{INTRODUCTION}

Tissue expander/implant-based reconstruction has been the most commonly used procedure for breast reconstruction after mastectomy. Due to the recent innovation of acellular dermal matrix $(\mathrm{ADM})$, the deficit of inferolateral coverage for expander and poor expansion in the lower pole has been improved in the setting of prosthetic reconstruction [1]. Furthermore, reduction of postoperative pain by preserving the anterior serratus or rectus abdominis musculofascial flap, rapid expansion, and better aesthetic outcomes have also been achieved by the use of $\mathrm{ADM}$ [1]. Despite these potential benefits, there is a growing concern regarding the complications of $\mathrm{ADM}$ such as skin flap necrosis, seroma, or infection compared to non-ADM-assisted reconstruction. Specifically, with respect to seroma, meta-analysis studies have reported a four-fold increase in the incidence of seroma in $\mathrm{ADM}$ cohorts [2-4]. At present, there are various types of human ADMs available for breast reconstruction, which go through 
different manufacturing and packaging processes. CGCryoDerm (CGBio Co., Seongnam, Korea) was first introduced in 2010 and offers a different matrix preservation process for freezing without drying the preparation. Unlike AlloDerm (LifeCell Co., Branchburg, NJ, USA) and CGDerm (CGBio Co.), which are manufactured using a similar process, CGCryoDerm does not require lengthy rehydration and needs to be melted for only 3 minutes before application [5]. Additionally, from a theoretical perspective, CGCryoDerm which does not go through a drying process, has a more preserved dermal structure such as collagen fibers, elastin, and vascular scaffolds as well as more abundant growth factors for angiogenesis and recellularization $[5,6]$. These factors play a role as essential elements for incorporation and revascularization of adjacent host tissues. In addition, progression toward completion is highly associated with perioperative complications, especially seroma formation $[4,7]$. To the best our knowledge, the majority of studies previously published on the outcomes of ADM have involved freeze-drying using processed materials such as AlloDerm (LifeCell Co.) [8]. The main study objective was to evaluate freezing alone (CGCryoDerm, CGBio Co.) and freeze-drying processed (CGCryoDerm, CGBio Co.) $\mathrm{ADM}$ to determine whether any differences were present in early complication profiles. Early postoperative outcomes, specifically rates of seroma formation as well drainage period, were used as clinical endpoints for evaluation.

\section{METHODS}

\section{Patient selection and data collection}

A retrospective chart review was performed and patients who underwent $\mathrm{ADM}$-assisted tissue expander placement for two stage breast reconstruction by three plastic surgeons between January of 2013 and July of 2014 at Samsung Medical Center were included in the study. The authors included unilateral tissue expander placement because bilateral mastectomy is a confounding factor for drainage caused by decreased blood supply in the medial portion of the breasts due to disruption of internal thoracic perforators [9]. In addition, irradiated breasts were excluded because radiation compromises the vascularity of skin flaps and prevents incorporation of ADM into the adherent tissue and increases complications such as seroma or infection [8]. Because of a significantly lower incidence of overall complications in delayed reconstruction, delayed cases were also excluded to eliminate confounding effects [4].

In order to increase the power of comparability, the authors excluded AlloDerm (LifeCell Co.) and other types of ADM-assisted cases. Patients were divided into two groups based on the types of $\mathrm{ADM}$-assisted expander reconstruction. One group in- cluded CGDerm and the other CGCryoDerm. The authors collected the following data from patient medical records: age, body mass index, smoking, neo adjuvant chemotherapy, and intraoperative findings including mastectomy weight, extent of axillary lymph node dissection, saline fill volume, and ratio to expander size. In addition, the total amount of drainage and drainage period were also calculated. Complications were divided into four main categories and recorded as follows: seroma, hematoma, infection, and mastectomy skin flap necrosis. Seroma was defined as clinically significant fluid collection that required percutaneous aspiration or surgical procedures. Other complications including mastectomy skin flap necrosis, infection, and hematoma were defined as the need for revision, antibiotics, or hematoma evacuation, respectively.

\section{Treatment procedure}

All surgeons followed a similar surgical protocol. After mastectomy was performed by a general surgeon, the surgical field was again prepared and draped. A sub-pectoral dissection was then performed and the lateral and inferior borders of the pectoralis major muscle were released for the ADM sling. The breast pocket was then irrigated sufficiently with antibiotic solution containing $500 \mathrm{~mL}$ of normal saline mixed with $1 \mathrm{~g}$ of cefazolin, 80 $\mathrm{mg}$ of gentamicin, and $10 \%$ povidone solution. For the preparation of ADM, CGCryoDerm was melted in saline for 3 minutes, while CGDerm was melted and rehydrated in saline for $20 \mathrm{~min}$ utes. Then, $\mathrm{ADM}$ was sutured from the inferomedial to the lateral corner of the pectoralis major muscle under the edge of the expander to adequately compartmentalize and prevent lateral migration. All patients had Mentor Tissue Expanders (Mentor, Santa Barbara, CA, USA) inserted. Two separate drains were used, one from cephalad toward the axillary area within the supra-pectoral pocket and the other along the inframammary fold within the sub-ADM pocket. The expander was then filled with saline as much as possible until the dead space of the pocket was filled but still preserved the microvascular circulation of the underlying the native mastectomy skin flap. Postoperatively, drains were maintained in place for approximately 1 to 2 weeks, depending on the volume of drainage. Next, drains were removed when the volume of drainage was less than $30 \mathrm{~mL}$ per 24 hours. Postoperative intravenous antibiotics were continued until the drains were removed.

\section{Statistical analysis}

Data were summarized using categorical data as percentages and continuous data as means with standard deviation. Comparison of CGDerm and CGCryoDerm-assisted groups was performed using the Fisher's exact test to evaluate the association between 
categorical variables and the two sample t-test for continuous variables. P-values less than 0.05 were considered statistically significant. Statistical analysis was performed using IBM SPSS ver. 19.0 (IBM Co., Armonk, NY, USA).

\section{RESULTS}

A total of 82 consecutive patients were included in the study and divided into two assisted tissue expander groups after total mastectomy: CGDerm $(\mathrm{n}=49,59.8 \%)$ and CGCryoDerm $(\mathrm{n}=33,40.9 \%)$. All patients were offered ADM immediately as an adjunct to their reconstruction. By analyzing the preoperative demographics between the CGDerm and CGCryoDerm groups, patients were found to be statistically similar in age (42.31 years and 42.64 years, respectively; $\mathrm{P}=0.857$ ), body mass index $\left(21.98 \mathrm{~m}^{2} / \mathrm{kg}\right.$ and $22.08 \mathrm{~m}^{2} / \mathrm{kg}$, respectively; $\left.\mathrm{P}=0.153\right)$, smoking status ( $10.2 \%$ and $6.1 \%$, respectively; $\mathrm{P}=0.510)$, and history of neo adjuvant chemotherapy ( $4.1 \%$ and $3.0 \%$, respectively; $\mathrm{P}=0.804)$ (Table 1). There was no difference of distribution in surgeons between two groups. For the intraoperative data, the incidence of axillary lymph node dissection, which disrupts the lymphatics and can affect drainage and the development of seroma, was higher in the CGCryoDerm group. However, there was no statistical significance between the two groups $(28.6 \%$

\section{Table 1. Comparison of patient demographics}

\begin{tabular}{|lccc|}
\hline Characteristic & $\begin{array}{c}\text { CGDerm } \\
(\mathbf{n}=\mathbf{4 9})\end{array}$ & $\begin{array}{c}\text { CGCryoDerm } \\
(\mathbf{n}=33)\end{array}$ & P-value \\
\hline Age $(\mathrm{yr})$ & $42.31 \pm 7.96$ & $42.64 \pm 8.37$ & 0.857 \\
Body mass index $\left(\mathrm{kg} / \mathrm{m}^{2}\right)$ & $21.98 \pm 2.96$ & $22.08 \pm 2.41$ & 0.153 \\
$\begin{array}{l}\text { Neo adjuvant } \\
\text { chemotherapy }\end{array}$ & $2(4.1)$ & $1(3.0)$ & 0.804 \\
Smoking & $5(10.2)$ & $2(6.1)$ & 0.510 \\
$\begin{array}{l}\text { Breast cancer staging } \\
0\end{array}$ & $6(12.2)$ & $8(24.2)$ & 0.484 \\
1 & $24(49.0)$ & $13(39.4)$ & \\
2 & $16(32.7)$ & $11(33.3)$ & \\
3 & $3(6.1)$ & $1(3.0)$ & \\
\hline Values are presented as mean \pm standard deviation or number (\%). & \\
\hline
\end{tabular}

and $36.4 \%$, respectively; $\mathrm{P}=0.457$ ). Additionally, the two groups showed similar mastectomy weight (363.06 g and $347.48 \mathrm{~g}$, respectively; $\mathrm{P}=0.692$ ) and percentage of expander fill volume (53.54\% and 52.86\%, respectively; $\mathrm{P}=0.877$ ) (Table 2). Regarding perioperative complications, the CGCryoDerm group had much lower rates of seroma when compared to the CGDerm group, but this result failed to achieve statistical significance at $3.0 \%$ vs. $10.2 \%(\mathrm{P}=0.221)$, respectively. Other complications were similar in both groups with incidence rates of $2.0 \%$ and $3.0 \%$ $(\mathrm{P}=0.776)$ for hematoma and infection, respectively, and $8.2 \%$ and $9.1 \%(\mathrm{P}=0.883)$ for mastectomy skin flap necrosis requiring wound revision, respectively. The incidence of total complications was slightly lower in the CGCryoDerm group but there was no significant difference between the two groups (Table 3 ). The trend for outcomes of drainage period and total amount was also explored. The total amount of drainage ( $921.24 \mathrm{~mL}$ vs. 992.97 $\mathrm{mL}$ ) until drain removal was slightly higher in the CGCryoDerm group when compared to the CGDerm group but there was no statistical significance with this finding. Finally, reconstructions with CGCryoDerm were found to have a significantly longer period of drainage when compared to reconstructions with CGDerm (11.91 days vs. 10.41 days; $\mathrm{P}=0.043$ ) (Table 4).

\section{DISCUSSION}

Evidence shows that the use of ADM improves definition and support of the infra-mammary fold, shields the expander from the overlying mastectomy skin flaps, and may reduce inflammatory changes that lead to the development of capsular contrac-

Table 2. Intraoperative factors between the two groups

\begin{tabular}{|lccc|}
\hline Characteristic & $\begin{array}{c}\text { CGDerm } \\
(\mathbf{n}=\mathbf{4 9})\end{array}$ & $\begin{array}{c}\text { CGCryoDerm } \\
(\mathbf{n = 3 3 )}\end{array}$ & P-value \\
\hline Mastectomy weight $(\mathrm{g})$ & $363.06 \pm 192.92$ & $347.48 \pm 141.31$ & 0.692 \\
$\begin{array}{c}\text { Axillary lymph node } \\
\text { dissection }\end{array}$ & $14(28.6)$ & $12(36.4)$ & 0.457 \\
$\begin{array}{c}\text { Percentage of tissue ex- } \\
\text { pander fill volume (\%) }\end{array}$ & $53.54 \pm 21.09$ & $52.86 \pm 16.71$ & 0.877 \\
\hline
\end{tabular}

\section{Table 3. Univariate analysis of complications between the two groups}

\begin{tabular}{|c|c|c|c|c|}
\hline Variable & CGDerm $(n=49)$ & CGCryoDerm $(n=33)$ & Odds ratio ( $95 \%$ confidence interval) & P-value \\
\hline Total complications ${ }^{\text {a) }}$ & $8(16.3)$ & $5(15.2)$ & $0.915(0.271-3.088)$ & 0.886 \\
\hline Seroma & $5(10.2)$ & $1(3.0)$ & $0.275(0.031-2.469)$ & 0.221 \\
\hline Hematoma & $1(2.0)$ & $1(3.0)$ & $1.500(0.091-24.857)$ & 0.776 \\
\hline Infection & $1(2.0)$ & $1(3.0)$ & $1.500(0.091-24.857)$ & 0.776 \\
\hline Mastectomy skin flap necrosis & $4(8.2)$ & $3(9.1)$ & $1.125(0.235-5.389)$ & 0.883 \\
\hline Total & 49 & 33 & - & - \\
\hline
\end{tabular}


Table 4. Outcomes of drainages between the two groups

\begin{tabular}{|lccc|}
\hline Variable & $\begin{array}{c}\text { CGDerm } \\
(\mathbf{n}=\mathbf{4 9})\end{array}$ & $\begin{array}{c}\text { CGCryoDerm } \\
(\mathbf{n}=\mathbf{3 3})\end{array}$ & P-value \\
\hline $\begin{array}{c}\text { Total amount of drainage } \\
(\mathrm{mL})\end{array}$ & $921.24 \pm 310.29$ & $992.97 \pm 457.32$ & 0.400 \\
Period of drainage (day) & $10.41 \pm 2.92$ & $11.91 \pm 3.66$ & 0.043 \\
\hline
\end{tabular}

ture. However, the higher incidence of complications, especially seroma, has still been a concern in several studies [8,10-16]. Michelotti et al. [14] reported an incidence rate of $6.3 \%$ for seroma complications in their study of $284 \mathrm{ADM}$-assisted expanders compared to $1.56 \%$ in the non-ADM group. Similarly, Chun et al. [16] published a large study of 269 immediate breast reconstructions using $\mathrm{ADM}$ and reported a 9.7\% seroma rate compared to $3.0 \%$ in the non- $\mathrm{ADM}$ group $(\mathrm{P}=0.01)$. In addition, Chun et al. [16] reported that ADMs increased the odds of seroma formation by 4.24 times $(\mathrm{P}=0.018)$. The majority of these studies involved AlloDerm (LifeCell Co.) although there are various types of $\mathrm{ADMs}$ that have been recently introduced such as DermaMatrix (Synthes Inc., West Chester, PA, USA), FlexHD (Ethicon Inc., Somerville, NJ, USA), AlloMax (Davol Inc., Warwick, RI, USA), CGDerm, and CGCryoDerm (CGBio Co.) [14]. All of these allogeneic skin products are produced using a similar protocol based on three steps including the following: (1) de-epithelization, (2) removal of cellular and immunogenic components leaving behind the basement membrane and cellular matrix, and (3) matrix preservation for supporting tissue integration and promoting vascularization. However, there are subtle differences in the manufacturing process of these ADMs depending on the source of tissue material, manufacturing methods, storage and surgical preparation, and cost [5]. In 2010, CGCryoDerm was introduced as the first allograft skin product processed using a cryopreserved method in the matrix preservation step to minimize damage to the 3-dimensional dermal structure. CGDerm and CGCryoDerm are similar in terms of their constituents and are made by the same company, but the method used in the third matrix preservation step results in different preparation and handling characteristics. CGDerm generally undergoes a freezing-drying process like other $\mathrm{ADMs}$, whereas CGCryoDerm is frozen at a temperature of $-40^{\circ} \mathrm{C}$ in the third step. Rehydration of CGDerm requires at least 20 minutes in normal saline, whereas CGCryoDerm requires only 3 minutes for melting without rehydration. Furthermore, the optimized cryoprotectant method used for CGCryoDerm preserves dermal contents including collagen type IV and results in a more abundant titer of growth factors and cytokines for angiogenesis. In the traditional freeze-drying process for $\mathrm{ADMs}$ such as AlloDerm or CGDerm, ice crystals inevitably form and cause primary destruction of the dermal matrix. In the subsequent drying process, the dermal structure is deformed more severely and tensile strength is weakened due to the space caused by water evaporation. In histologic studies, better engraftment of CGCryoDerm has been confirmed by a higher alpha smooth muscle actin ratio to total cells when compared to CGDerm [6]. At our institution, CGCryoderm is one of the most used ADM materials for lower pole coverage along with CGDerm and AlloDerm and these three products have been used interchangeably. CGCryoDerm, with a different processing step (i.e., cryopreserved product), has been widely used, however, few studies have been published documenting its clinical outcome in breast reconstruction. Before analysis, the two groups in the present study were well matched in terms of age, body mass index, smoking, mastectomy weight, extent of lymph node dissection, and distribution of surgeons, all of which can affect complications and skew results if not controlled for. The results of the current study showed that there is no demonstrable difference in overall complications between the CGCryoDerm and CGDerm groups. With regards to the complication of seroma, it is known that seroma formation in the ADM-assisted expander might be caused by increased dead space due to a mismatch between the skin envelope and underlying expander before complete revascularization and incorporation of the $\mathrm{ADM}$, which takes approximately 2 weeks as confirmed in a previous animal study $[4,7]$. We believed it was reasonable to hypothesize that CGCryoDerm has a better regenerative potential for incorporation and thus can lower seroma complication rates and have shorter drain periods when compared to CGDerm. According our results, CGCryoDerm had a lower incidence of seroma formation $(n=1 / 33,3 \%)$ than the CGDerm group $(n=5 / 49$, $10.2 \%)$, but this difference was not statistically significant. Lee et al. [5] compared AlloDerm and CGCryoDerm groups for the presence of complications including seroma, infection, skin flap necrosis, capsular contracture, and implant loss during immediate breast reconstruction. In their study, the authors found no difference in clinically significant complications between the two groups, but the incidence of seroma was much lower in the CGCryoDerm group. This result is consistent with the findings in our study. The authors suggested the possibility that the small sample size in our study underpowered the statistical significance of differences in seroma rates between the two groups and that larger studies may be required. Until complete incorporation of the ADM in the mastectomy skin flap occurs, proper drain placement is essential for minimizing fluid accumulation to provide an environment of negative pressure and to ensure contact between the ADM and mastectomy skin flap [8]. The authors found that CGCryoDerm had a longer drainage period 
(average of 1.5 days longer) when applying the same drainage protocol ( $<30 \mathrm{~mL}$ for 24 hours). This result seems to be paradoxical to the hypothesis that CGCryoDerm has a shorter drain period. Although the reason for this result is not clear, we recommend that surgeons may consider longer drainage periods when using CGCryoderm. Anecdotally, there are also differences between the two products with respect to the methods used for manipulating them during surgery. CGCryoDerm is felt to be more pliable and easier to handle and shape into the inferolateral curvature of the breast compared to CGDerm or AlloDerm. Taking into account the similar cost of both products and similar complication rates including seroma, this study suggests that the choice between these two products can be based on surgeon preference, given the equivalency of the products. The authors recognize that this study is a preliminary study of CGCryoDerm and therefore has limitations because of the small sample size and relatively short follow-up period. Unfortunately, our data were not powered sufficiently to determine whether the use of CGCryoDerm resulted in more favorable complication rates and long-term outcomes. To identify the long-term effect of better incorporation characteristics in CGCryoderm, further investigation regarding outcomes of CGCryoderm should be analyzed after permanent implant surgery. In the future, a larger, randomized, prospective study should be performed to answer these important questions.

This study demonstrated no significant differences in the rate of overall complications, including seroma formation, between the two experimental groups. The incidence of seroma in the CGCryoderm group was much lower than the CGDerm group, although this finding did not have statistical significance. Preliminary findings indicate no significant difference between expander-based reconstructions using CGCryoderm and those using CGDerm.

\section{REFERENCES}

1. Martin L, O’Donoghue JM, Horgan K, et al. Acellular dermal matrix (ADM) assisted breast reconstruction procedures: joint guidelines from the Association of Breast Surgery and the British Association of Plastic, Reconstructive and Aesthetic Surgeons. Eur J Surg Oncol 2013;39:425-9.

2. Spear SL, Majidian A. Immediate breast reconstruction in two stages using textured, integrated-valve tissue expanders and breast implants: a retrospective review of 171 consecutive breast reconstructions from 1989 to 1996. Plast Reconstr Surg 1998;101:53-63.

3. Cordeiro PG, McCarthy CM. A single surgeon's 12-year experience with tissue expander/implant breast reconstruc- tion: part I. A prospective analysis of early complications. Plast Reconstr Surg 2006; 118:825-31.

4. Ho G, Nguyen TJ, Shahabi A, et al. A systematic review and meta-analysis of complications associated with acellular dermal matrix-assisted breast reconstruction. Ann Plast Surg 2012;68:346-56.

5. Lee JH, Park KR, Kim TG, et al. A comparative study of CG CryoDerm and AlloDerm in direct-to-implant immediate breast reconstruction. Arch Plast Surg 2013;40:374-9.

6. CGBio. Dentistry: CGCryoDerm [Internet]. Sungnam: CGBio Co.; [cited 2014 Dec 2]. Available from: http://cgbio.co.kr/sub2_4_2.

7. Wong AK, Schonmeyr B, Singh P, et al. Histologic analysis of angiogenesis and lymphangiogenesis in acellular human dermis. Plast Reconstr Surg 2008;121:1144-52.

8. Nahabedian MY. Acellular dermal matrices in primary breast reconstruction: principles, concepts, and indications. Plast Reconstr Surg 2012;130:44S-53S.

9. Antony AK, McCarthy CM, Cordeiro PG, et al. Acellular human dermis implantation in 153 immediate two-stage tissue expander breast reconstructions: determining the incidence and significant predictors of complications. Plast Reconstr Surg 2010;125:1606-14.

10. Weichman KE, Wilson SC, Weinstein AL, et al. The use of acellular dermal matrix in immediate two-stage tissue expander breast reconstruction. Plast Reconstr Surg 2012;129:1049-58.

11. Venturi ML, Mesbahi AN, Boehmler JH 4th, et al. Evaluating sterile human acellular dermal matrix in immediate expander-based breast reconstruction: a multicenter, prospective, cohort study. Plast Reconstr Surg 2013;131:9e-18e.

12. Vardanian AJ, Clayton JL, Roostaeian J, et al. Comparison of implant-based immediate breast reconstruction with and without acellular dermal matrix. Plast Reconstr Surg 2011; 128:403e-410e.

13. Sbitany H, Sandeen SN, Amalfi AN, et al. Acellular dermisassisted prosthetic breast reconstruction versus complete submuscular coverage: a head-to-head comparison of outcomes. Plast Reconstr Surg 2009; 124:1735-40.

14. Michelotti BF, Brooke S, Mesa J, et al. Analysis of clinically significant seroma formation in breast reconstruction using acellular dermal grafts. Ann Plast Surg 2013;71:274-7.

15. Liu AS, Kao HK, Reish RG, et al. Postoperative complications in prosthesis-based breast reconstruction using acellular dermal matrix. Plast Reconstr Surg 2011;127:1755-62.

16. Chun YS, Verma K, Rosen H, et al. Implant-based breast reconstruction using acellular dermal matrix and the risk of postoperative complications. Plast Reconstr Surg 2010;125: 429-36. 